\title{
Near-Infrared Emitting Colloidal PbS Nanoplatelets: Lateral Size Control and Optical Spectroscopy
}

\author{
Ali Hossain Khan, ${ }^{\dagger}{ }^{\dagger}$ Rosaria Brescia, ${ }^{\dagger}$ Anatolii Polovitsyn, ${ }^{\dagger, \S}$ Ilaria Angeloni, ${ }^{\dagger, \#}$ Beatriz Martín-García, \\ and Iwan Moreels $*, \dagger$ \\ ${ }^{\dagger}$ Istituto Italiano di Tecnologia, Via Morego 30, 16163 Genova, Italy \\ ${ }^{\S}$ Dipartimento di Fisica, Università degli Studi di Genova, Via Dodecaneso 33, 16146 Genova, Italy \\ \#Dipartimento di Chimica e Chimica Industriale, Università degli Studi di Genova, Via Dodecaneso 33, 16146 Genova, Italy
}

\section{Supporting Information}

\begin{abstract}
Two dimensional (2D) colloidal PbS nanoplatelets (NPLs) with a thickness of $1.8-2.8 \mathrm{~nm}$ have been synthesized using a single-molecule precursor approach with lead octadecylxanthate. The lateral dimensions were tuned by varying the reaction temperature, growth time, and capping ligands. Transmission electron microscopy and $\mathrm{X}$-ray diffraction reveal that the NPLs have an orthorhombic crystal structure rather than the rocksalt phase usually reported for bulk and nanostructured $\mathrm{PbS}$. The $1.8 \mathrm{~nm}$ thickness, in combination with the tunable lateral dimensions, results in a blue-shifted absorption peak at $715-730 \mathrm{~nm}$ and a $48-68 \mathrm{~nm}$ narrow emission spectrum with a surprisingly small, $18 \mathrm{~nm}$ Stokes shift at room temperature. The fluorescence lifetime of these $\mathrm{PbS}$

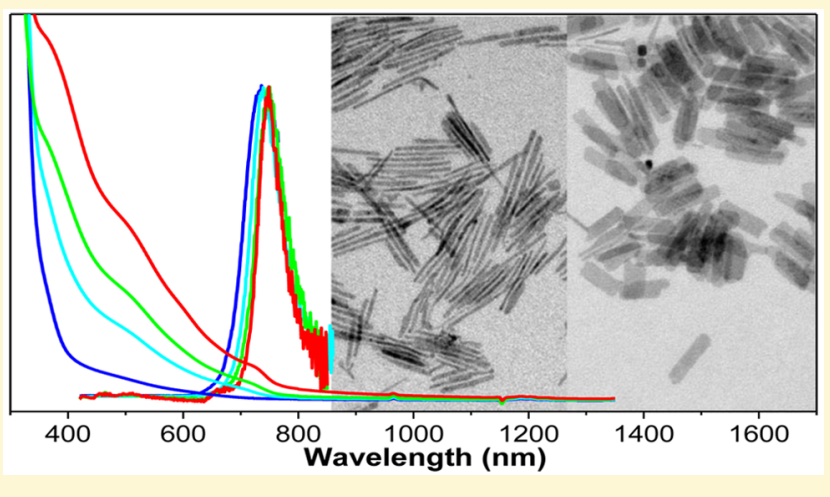
NPLs is 2 orders of magnitude shorter than the typical lifetime in $0 \mathrm{D}$ colloidal PbS quantum dots, highlighting the advantageous properties of colloidal $2 \mathrm{D}$ nanostructures that combine strong transversal with weak lateral confinement.
\end{abstract}

\section{INTRODUCTION}

In the field of colloidal nanocrystals (NCs), two dimensional (2D) nanoplatelets (NPLs) have recently appeared as a new class of NCs that share characteristics with epitaxial quantum wells, while maintaining the benefit of being suitable for solution-based processing. Several research groups have demonstrated that 2D NPLs form promising building blocks for novel photonic applications, such as single-particle electronic devices, ${ }^{1}$ color-saturated light emitting diodes with narrow electroluminescence spectrum, ${ }^{2}$ luminescence-based oxygen sensors, ${ }^{3}$ or low-threshold lasers. ${ }^{4-6}$ They combine strong confinement in one direction and thus tunable optical properties via the NPL thickness, with weakly bound exciton motion in the other two dimensions, yielding an enhanced band-edge oscillator strength. ${ }^{7}$ Shape control of colloidal NCs toward 2D NPLs results in unique spectroscopic properties, such as a short photoluminescence (PL) lifetime, ${ }^{8}$ high color purity, $^{2}$ a strongly reduced Stokes shift, ${ }^{9}$ exceptionally large exciton $^{7}$ and biexciton binding energies, ${ }^{6}$ and a suppressed Auger recombination. ${ }^{10}$ Since NPLs have a thickness that is controlled with atomic precision, almost no inhomogeneous $\mathrm{PL}$ line broadening is observed at room temperature. ${ }^{9}$ An added benefit that strongly encourages the development of colloidal NPLs is that the carrier confinement and exciton binding energies are much stronger in these systems than in epitaxial quantum wells, since they are capped with organic ligands and typically surrounded by low-dielectric media, further boosting the giant-oscillator strength effect via increased dielectric confinement. ${ }^{7,8}$

In 2006, the Hyeon group reported the first colloidal synthesis of ultrathin 2D CdSe nanoribbons. ${ }^{11}$ Thereafter, there have been several publications on progress in the synthesis of various $2 \mathrm{D} \mathrm{CdE}(\mathrm{E}=\mathrm{S}, \mathrm{Se}, \mathrm{Te}) \mathrm{NPLs}$, in particular in the control of crystal structure, ${ }^{12}$ half-integer thickness and a cation-rich surface, ${ }^{13}$ and core $/$ shell $^{14}$ and core $/$ crown $^{15}$ structures. Much of the work to date on 2D NPLs has focused on materials design for the visible spectral region. However, the study of NPLs with efficient NIR emission is essential to expand the application of $2 \mathrm{D}$ materials into this technologically important spectral region. In this sense, OD lead chalcogenide quantum dots (QDs) are well-known as NIR emitting materials. ${ }^{16}$ In 2010, Weller and co-workers first reported the synthesis of $2 \mathrm{D} \mathrm{PbS}$ nanosheets with lateral dimensions of several hundred nanometers, obtained via oriented attachment of PbS NCs. ${ }^{17}$ While transistor ${ }^{18}$ and photovoltaic ${ }^{19}$ devices have already been reported, at present, limited data exist on the $\mathrm{PbS}$ NPL fluorescence properties. ${ }^{20-23}$ Most likely, the specific growth conditions that lead to these large NPLs also introduce

Received: December 1, 2016

Revised: $\quad$ March 4, 2017

Published: March 6, 2017 

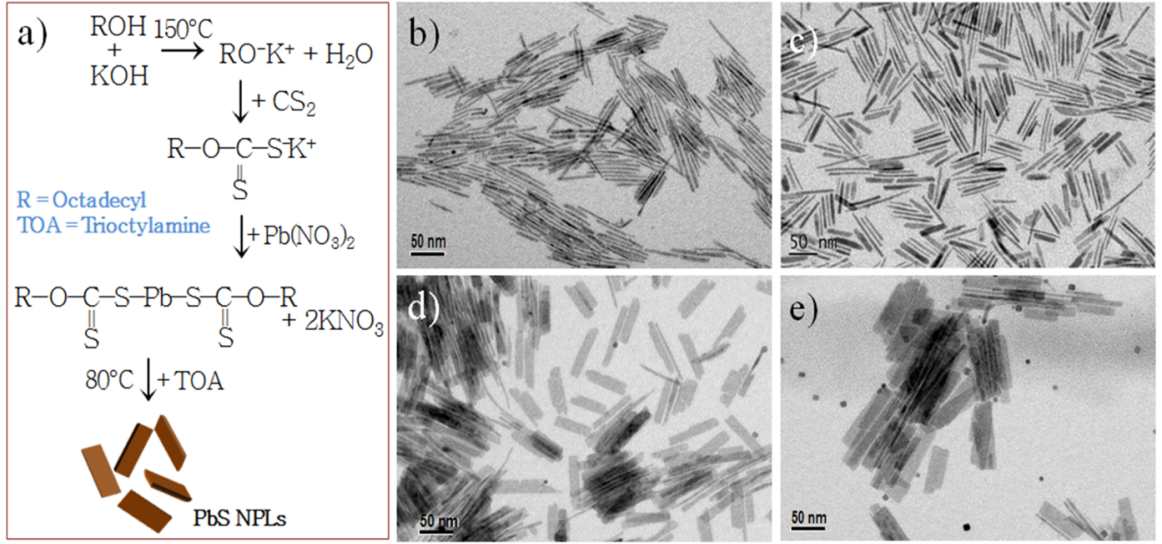

Figure 1. (a) Scheme illustrating the formation mechanism of PbOctdX and PbS NPLs. BF-TEM images of PbS NPLs prepared by annealing at 80 ${ }^{\circ} \mathrm{C}$ for (b) $10 \mathrm{~min}$, (c) $40 \mathrm{~min}$, (d) $2 \mathrm{~h}$, and (e) $5 \mathrm{~h}$.

defects that reduce the PL, so further progress in the NPL growth and understanding of their optoelectronic properties is still required to enable the development of NPL-based photonic applications in the NIR. ${ }^{24}$

Here, we report on the synthesis of PbS NPLs, with a significantly improved control over the final size and shape of the NPLs, using a single-source precursor route that is performed at temperatures of only $80-90{ }^{\circ} \mathrm{C}$. The formation and growth of the NPLs were monitored at different stages of the reaction by transmission electron microscopy (TEM) and optical spectroscopy. The length (from 23 to $167 \mathrm{~nm}$ ) and width (from 3.5 to $27 \mathrm{~nm}$ ) can be tuned by varying the reaction temperature, growth time, precursor concentration, and capping ligands. The crystal structure of the NPLs was investigated in detail by X-ray and selected-area electron diffraction (XRD and SAED, respectively) and high resolution TEM (HR-TEM) analysis, which agreed on the NPL crystal structure matching with an orthorhombic $\mathrm{PbS}$ phase rather than with the more often occurring cubic PbS phase. TEM images of vertically standing PbS NPLs revealed a thickness of about $2 \mathrm{~nm}$, which explains the strongly blue-shifted band gap. The emission spectra are very narrow (fwhm of $48-68 \mathrm{~nm}$; 106-156 meV) compared to spherical PbS QDs that have a band gap at similar energies, with almost no Stokes shift. Both are typical characteristics of 2D NPLs. A tunable PL from 735 to $748 \mathrm{~nm}$ was observed for different lateral extensions, with emission lifetimes of 10 to $60 \mathrm{~ns}$, up to 2 orders of magnitude faster than spherical PbS QDs.

\section{EXPERIMENTAL SECTION}

Materials. Octadecanol (99\%), potassium hydroxide (90\%), carbon disulfide $(\geq 99.9 \%)$, lead nitrate $(\geq 99.0 \%)$, lead acetate trihydrate $(99.999 \%)$, lead chloride $(99.999 \%)$, trioctylamine (TOA; 98\%), triethylamine (TEA; 99\%), tridodecylamine (TDDA; 97\%), octylamine (99\%), methanol (99.9\%), ethanol ( $\geq 99.8 \%)$, acetone $(\geq 99.8 \%)$, toluene $(99.8 \%)$, oleic acid $(90 \%)$, 1,2-dichlorobenzene (99\%), acetonitrile (99.8\%), chloroform (99.8\%), and tetrachloroethylene (99\%) were purchased from Sigma-Aldrich. All chemicals were used without further purification.

Synthesis of Lead Octadecylxanthate (PbOctdX). First, the alkoxide salt was generated at $150^{\circ} \mathrm{C}$ by reacting potassium hydroxide with octadecanol (1:1 molar ratio) for about $30 \mathrm{~min}$ in air. The reaction flask was then cooled to room temperature with an air flow, and carbon disulfide $\left(\mathrm{CS}_{2} /\right.$ alkoxide molar ratio of 1.2:1) was injected swiftly. The alkoxide moiety reacts with carbon disulfide to give potassium octadecylxanthate. Afterward, toluene was added to dissolve and remove the residual raw material. The precipitated product was then dried in a freeze-dryer. PbOctdX was prepared by slowly adding an equimolar amount of an aqueous solution of lead nitrate to the potassium octadecylxanthate dispersed in water, with continuous stirring. The precipitate was separated by filtration and washed several times with Milli-Q water.

PbS NPL Synthesis. In a three-neck flask, $90 \mathrm{mg}(0.1 \mathrm{mmol})$ of PbOctdX powder and $5 \mathrm{~mL}$ of TOA were mixed and degassed under vacuum for $30 \mathrm{~min}$ at room temperature. The reaction mixture was then slowly heated to $80{ }^{\circ} \mathrm{C}$ under argon flow. At this temperature, $\mathrm{PbOctdX}$ decomposes into $\mathrm{PbS}$ according to the Chugaev reaction (Figure S1). ${ }^{25}$ The final temperature was reached after $15 \mathrm{~min}$. The reaction was kept at the growth temperature for up to $20 \mathrm{~h}$. With increasing growth time, the color of the reaction turned from orange to red and finally to dark red. After the completion of the reaction, the flask was cooled to room temperature. After synthesis, the crude sample was first diluted with $2 \mathrm{~mL}$ of toluene and $2 \mathrm{~mL}$ of oleic acid. Then, the NPLs were separated from the unreacted xanthate by adding $2 \mathrm{~mL}$ of 1,2-dichlorobenzene, followed by precipitation with acetonitrile and centrifugation at $6000 \mathrm{rpm}$ for $5 \mathrm{~min}$. We observed that both oleic acid and 1,2-dichlorobenzene improve the dissolution of the unreacted xanthates. After centrifugation, the NPLs were resuspended in toluene. A second precipitation was performed using acetonitrile. All purification processes were done under inert atmosphere. Finally, the purified NPLs were redispersed into tetrachloroethylene or chloroform.

Transmission Electron Microscopy. Samples were prepared by drop casting dilute NPL suspensions onto carbon coated copper grids. Bright field TEM (BF-TEM) images were acquired on a JEOL JEM1011 microscope equipped with a thermionic gun operated at $100 \mathrm{kV}$ accelerating voltage. For HR-TEM, samples were prepared by drop casting dilute NPL suspensions onto 400 mesh copper grids that were coated with a holey carbon/ultrathin carbon film. HR-TEM measurements were carried out on a JEOL JEM-2200FS microscope equipped with a Schottky emitter operated at $200 \mathrm{kV}$, a CEOS spherical aberration corrector for the objective lens, and an in-column energy filter (Omega-type). Selected area electron diffraction (SAED) patterns were acquired at constant camera length with the sample placed at the eucentric height and after carefully focusing the NC images. The camera length was calibrated using a nanocrystalline Au sputtered film on a standard carbon-covered $\mathrm{Cu}$ grid. The elaboration of SAED patterns (beam-stop removal, centering, azimuthal integration, and background subtraction) was carried out using PASAD. ${ }^{26}$

XRD Measurements. XRD analysis was performed on a PANanalytical Empyrean X-ray diffractometer equipped with a 1.8 $\mathrm{kW} \mathrm{Cu} \mathrm{K \alpha}$ ceramic X-ray tube and PIXcel ${ }^{3 \mathrm{D}} 2 \times 2$ area detector and operating at $45 \mathrm{kV}$ and $40 \mathrm{~mA}$. Samples for the XRD measurements were prepared in a glovebox by dropping a concentrated NPL dispersion onto a miscut silicon substrate. The diffraction patterns 

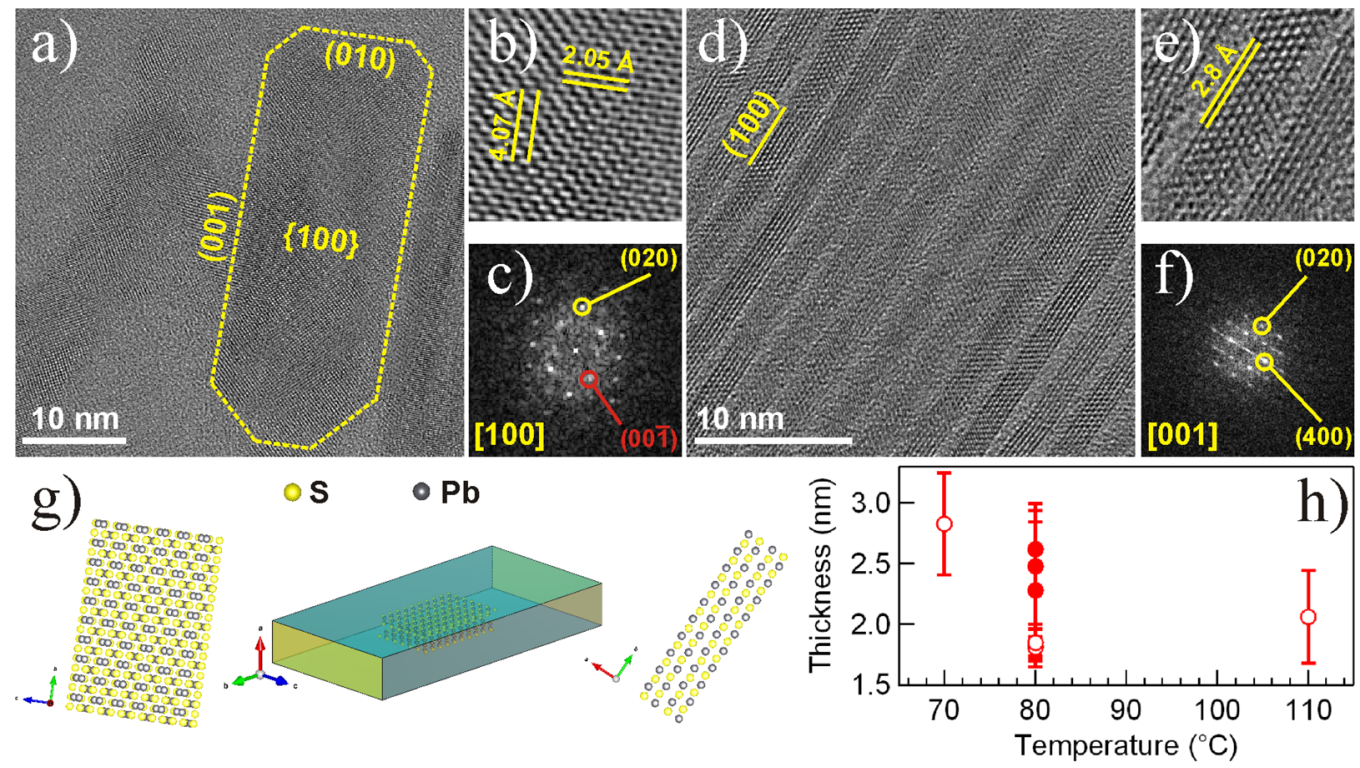

Figure 2. (a) HR-TEM image from a single PbS NPL lying with the extended facet on the support film ( $5 \mathrm{~h}$ reaction time). A portion of the NPL is

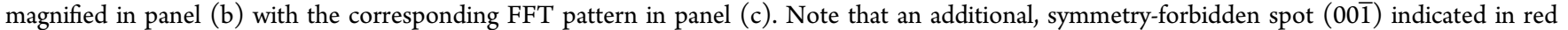
appears in the FFT, suggesting the presence of anisotropic lattice distortions. (d) HR-TEM image of vertically standing PbS NPLs in a stack, with magnified region in panel (e) and corresponding FFT in panel (f). (g) Schematic of the structure and faceting of the NPLs, with corresponding planview and cross-section, as derived from HR-TEM images shown in the corresponding panels above ( $\mathrm{S}$ atoms yellow, $\mathrm{Pb}$ atoms are gray). (h) Variation of the thickness for NPL samples prepared at different temperatures and growth time. Filled symbols represent aliquots of a synthesis at 80 ${ }^{\circ} \mathrm{C}$. Open symbols are different synthesis batches.

were collected using Parallel-Beam (PB) geometry and symmetric reflection mode.

Optical Characterization. Absorbance spectra were recorded using a Varian Cary 5000 UV-vis-NIR spectrophotometer. Steadystate and time-resolved PL emission spectra were measured using an Edinburgh Instruments FLS920 spectrofluorometer. The steady-state PL was collected by exciting the samples at $400 \mathrm{~nm}$ with a xenon lamp. The PL decay traces were recorded by exciting the samples at $405 \mathrm{~nm}$ using a 50 ps laser diode at a repetition rate of $0.05-1 \mathrm{MHz}$, to ensure complete decay of the emission between the excitation pulses. The data were collected at the PL peak position with an emission bandwidth of $10 \mathrm{~nm}$.

\section{RESULTS AND DISCUSSION}

Single-source precursors such as metal xanthates present a unique avenue to produce $2 \mathrm{D}$ metal sulfide semiconductor nanocrystals. ${ }^{27-30}$ Through a slow decomposition in longchained amine solvents at relatively low temperature (typically $70-130{ }^{\circ} \mathrm{C}$ ), anisotropic growth is promoted, ${ }^{27-30}$ and after several hours, well-defined 2D nanocrystals are generated (Figure 1). The lamellar structure of the ligands likely plays an important role to control the shape of the nanostructure, inducing a templated growth through attachment of small clusters or monomers to the edges of the initially nucleated $\mathrm{PbS}$ NPLs. ${ }^{31}$ Here, size-controlled PbS NPLs have been synthesized by decomposing lead octadecylxanthate (PbOctdX) into TOA, with $\mathrm{PbOctdX}$ prepared from the reaction of potassium octadecylxanthate with lead nitrate (Figure 1a).

In a typical synthesis, NPLs were grown at $80{ }^{\circ} \mathrm{C}$ under argon flow for $10 \mathrm{~min}$ to $5 \mathrm{~h}$ (see Experimental Section for details). The slow NPL growth in this synthesis route allows for a convenient tuning of the size via the reaction time. ${ }^{29}$ The BFTEM images of aliquots grown for $10 \mathrm{~min}, 40 \mathrm{~min}, 2 \mathrm{~h}$, and $5 \mathrm{~h}$ are presented in Figure $1 \mathrm{~b}-\mathrm{e}$. NPLs of $3.5 \mathrm{~nm}$ in width and 48 $\mathrm{nm}$ in length (Figure $1 \mathrm{~b}$ ) evolved into $21 \mathrm{~nm}$ wide and $83 \mathrm{~nm}$ long NPLs (Figure 1e) when synthesis time progressed from 10 min to $5 \mathrm{~h}$. Ten hours after the start of the reaction, PbS NPLs with a length of more than $100 \mathrm{~nm}$ are observed (Figure S2a, Table S1). However, prolonged synthesis times also resulted in the conucleation and growth of $8.6 \mathrm{~nm}$ diameter $\mathrm{PbS}$ cubes (Figure S2b). The high aspect ratio, from 13.7:1 at $10 \mathrm{~min}$ to 4.0:1 after 5 h, suggests a kinetic growth regime, where initially a fast growth along one direction is promoted.

HR-TEM and corresponding Fast Fourier Transform (FFT) images of a single NPL (aliquot taken at $5 \mathrm{~h}$ ) show a single crystalline structure that matches with orthorhombic $\mathrm{PbS}$ (ICSD 648451, $a=11.28 \AA, b=4.02 \AA, c=4.29 \AA$ ) rather than with the cubic phase assigned to $2 \mathrm{D} \mathrm{PbS}$ nanocrystals (Figure 2). ${ }^{17,29}$ The interplane distances of $2.8,2.04$, and $2.05 \AA$, respectively, agree well with the values for orthorhombic $\mathrm{PbS}$ and are different from the expected distance of $3 \AA$ for cubic $\mathrm{PbS}$. The extended facets of the NPLs are formed by $\{100\}$ planes, with $\{010\}$ and $\{001\}$ edge facets (Figure 2a), as also shown in the scheme (Figure $2 \mathrm{~g}$, drawings created by VESTA). ${ }^{32}$ HR-TEM images of NPLs standing on their side reveal a uniform thickness of $\sim 1.8 \mathrm{~nm}$ corresponding to 7 atomic layers along the [001] direction (Figure 2d). A further investigation of the thickness was performed for samples prepared at different temperatures and growth time (Figure S3, Table S2, Figure $2 \mathrm{~h}$ ). When taking aliquots during a synthesis at $80{ }^{\circ} \mathrm{C}$, no significant increase in thickness was observed between samples taken at $40 \mathrm{~min}, 5 \mathrm{~h}$, and $10 \mathrm{~h}$ (Figure $2 \mathrm{~h}$, filled symbols), confirming that the thickness is determined by the initial seeds. Although a slight decrease in thickness, of less than $1 \mathrm{~nm}$, is observed when increasing the synthesis temperature, this could also be due to batch-to-batch variation. Indeed, when repeating the synthesis at $80{ }^{\circ} \mathrm{C}$ (growth time of $5 \mathrm{~h}$ ), variations in thickness up to $0.5 \mathrm{~nm}$ (thicknesses ranging from 1.8 to $2.3 \mathrm{~nm}$ ) were observed.

SAED and powder XRD patterns of a sample grown for $5 \mathrm{~h}$ were compared with the calculated peak positions for cubic 
(ICSD 38293, $a=5.934 \AA$ ) and orthorhombic (ICSD 648451) $\mathrm{PbS}$ phases (Figures 3 and S4) and confirmed the HR-TEM
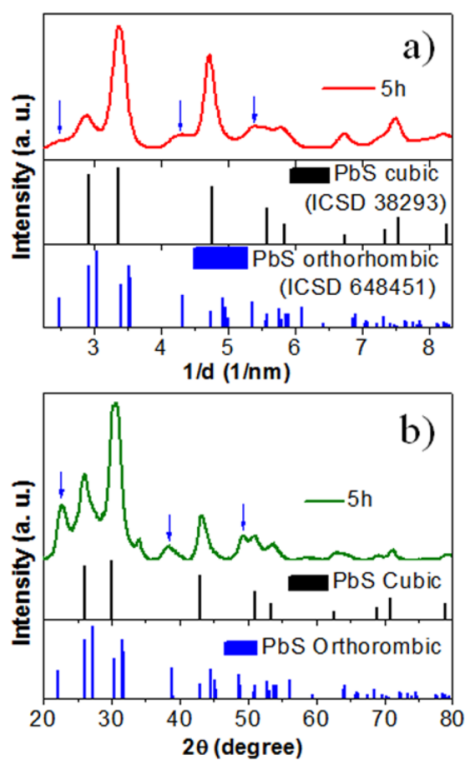

Figure 3. (a) Azimuthally integrated SAED patterns of the $5 \mathrm{~h}$ NPLs (grown at $80^{\circ} \mathrm{C}$ ), compared to database patterns of the two reference $\mathrm{PbS}$ phases. (b) XRD patterns of the same NPLs, again compared with standard patterns of cubic and orthorhombic $\mathrm{PbS}$.

results. In both SAED and XRD, additional peaks (indicated by arrows) can be associated with an orthorhombic crystal structure. Note that we could exclude the contribution of residual $\mathrm{PbOctdX}$, as this yields a different $\mathrm{XRD}$ pattern (Figure S5).

A possible reason for the observed lower symmetry in these highly confined 2D structures is the lack of periodicity along one out-of-plane direction. ${ }^{33,34}$ Moreover, while the transformation of bulk $\mathrm{PbS}$ from rocksalt to orthorhombic phase typically occurs at high pressure, ${ }^{35}$ it was recently shown that lead chalcogenide QDs undergo a lattice distortion already at room temperature and ambient pressure, ${ }^{36,37}$ with a displacement of the $\mathrm{Pb}$ sublattice driven by ligand-induced tensile strain at sizes below $8 \mathrm{~nm} .{ }^{37}$ A similar distortion may occur in $\mathrm{PbS}$ NPLs, giving rise to the observed XRD and SAED patterns associated with the orthorhombic crystal structure.

In addition to the synthesis time, we were also able to tune the shape and size of our PbS NPLs by the reaction temperature, precursors, and the organic ligands. The optimal temperature range for the NPLs synthesis was found to be $80-$ $90{ }^{\circ} \mathrm{C}$. At lower temperature (e.g., $70{ }^{\circ} \mathrm{C}$ ), PbOctdX decomposes very slowly in TOA, leading to only $8 \times 36 \mathrm{~nm}^{2}$ NPLs after $2 \mathrm{~h}$ (Figure S6). On the other hand, at temperatures above $90{ }^{\circ} \mathrm{C}$, they form highly anisotropic nanowires, and the final suspension also contains a significant amount of byproducts such as $\mathrm{PbS}$ nanocubes (Figure S6, Table S3). Likely, the templated growth ${ }^{31}$ is (partially) disrupted at higher temperatures, leading to a $3 \mathrm{D}$ growth.

TOA acts as a mild reducing agent of PbOctdX, ${ }^{28,38}$ and by using different amines, we were able to further tune the NPL shape. We observed that primary amines such as octylamine, dodecylamine, octadecylamine, and oleylamine react with PbOctdX already at room temperature and produce small particles and cubes at $80{ }^{\circ} \mathrm{C}$, and hence, they are not suitable for the NPL synthesis (Figure S7). A reduced reactivity of trialkyl amines however enables control over the lateral NPL dimensions via the alkyl chain length in reactions performed at $80{ }^{\circ} \mathrm{C}$. Triethylamine (TEA), for instance, produces $89 \times 12$ $\mathrm{nm}^{2} \mathrm{NPLs}$ after $2 \mathrm{~h}$ of reaction at $80{ }^{\circ} \mathrm{C}$ (Figure $4 \mathrm{a}$ ), whereas
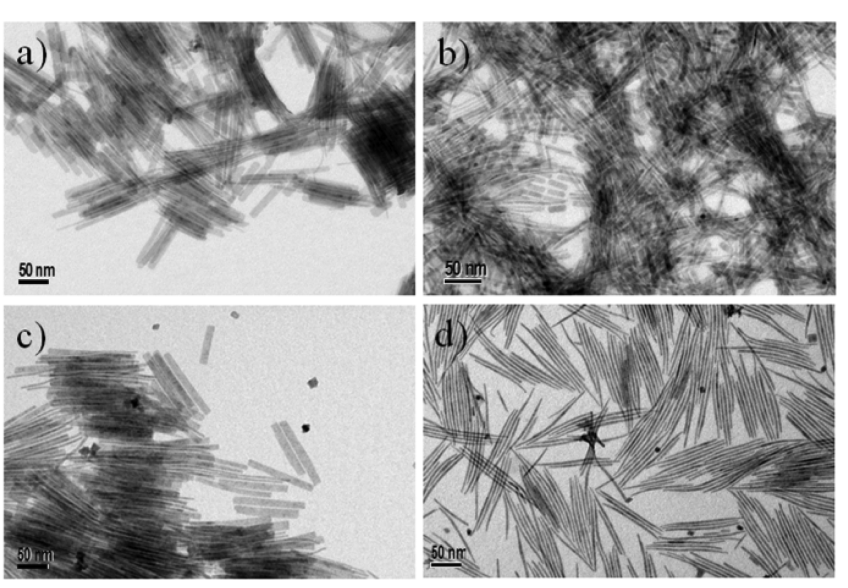

Figure 4. BF-TEM images of PbS NPLs prepared in (a) TEA, (b) TDDA, (c) TOA using $\mathrm{Pb}(\mathrm{OAc})_{2}$, and (d) TOA using $\mathrm{PbCl}_{2}$, for $2 \mathrm{~h}$ annealing at $80^{\circ} \mathrm{C}$.

tridodecylamine (TDDA) produces smaller, $26 \times 6 \mathrm{~nm}^{2}$ NPLs under the same conditions, however with thin nanowires as byproducts (Figure 4b). Larger NPLs can be obtained by adding lead acetate $\left(\mathrm{Pb}(\mathrm{OAc})_{2}, 0.1 \mathrm{mmol}\right)$ or lead chloride $\left(\mathrm{PbCl}_{2}, 0.1 \mathrm{mmol}\right)$ at the beginning of the reaction. These more reactive $\mathrm{Pb}$-precursors lead to uniform $118 \times 11 \mathrm{~nm}^{2} \mathrm{NPLs}$ and $123 \times 3.5 \mathrm{~nm}^{2}$ NPLs, respectively, after $2 \mathrm{~h}$ of growth (Figure $4 c, d)$.

Purification of the samples is a key step before the optical properties can be studied. Initially, we observed a strong contribution from Rayleigh scattering in the absorbance spectrum of the NPLs, due to the presence of unreacted PbOctdX (Figure S8). These xanthates are easily precipitated along with the NPLs by nonsolvents such as methanol, ethanol, acetone, or acetonitrile, and they are readily dispersible in nonpolar solvents such as chloroform, toluene, or tetrachloroethylene (TCE). Generally, PbOctdX remains as micron size flakes, with the NPLs embedded inside (Figure S9). For this reason, it is not possible to separate the NPLs from PbOctdX by simply adding the typical nonsolvents above. However, we observed that adding both oleic acid (OA) and dichlorobenzene (DCB) can dissolve the unreacted PbOctdX while keeping the NPLs intact (Figure S10). The OA-DCB treatment yields a clear solution after $5 \mathrm{~min}$, from which the NPLs can be precipitated by the addition of acetonitrile and centrifugation.

The absorbance spectra of the purified NPLs are shown in Figure 5a. First, while we observed minor absorption peaks in the NIR, these could be attributed to residual organics (Figure S11). After a second purification step, no significant features except for a small Rayleigh scattering background were detected up to $2000 \mathrm{~nm}$. More importantly, an absorption peak at 715 $\mathrm{nm}$ can clearly be observed already after $40 \mathrm{~min}$ of reaction time. This peak red shifts upon further NPL growth, reaching $730 \mathrm{~nm}$ after $5 \mathrm{~h}$. Additional transitions around 510 and 370 $\mathrm{nm}$ can be distinguished. The sharp rise below $400 \mathrm{~nm}$ for the smallest sample, grown for $10 \mathrm{~min}$, is due to some residual $\mathrm{PbOctdX}$ (Figure S11), since the decomposition of PbOctdX is very slow. 

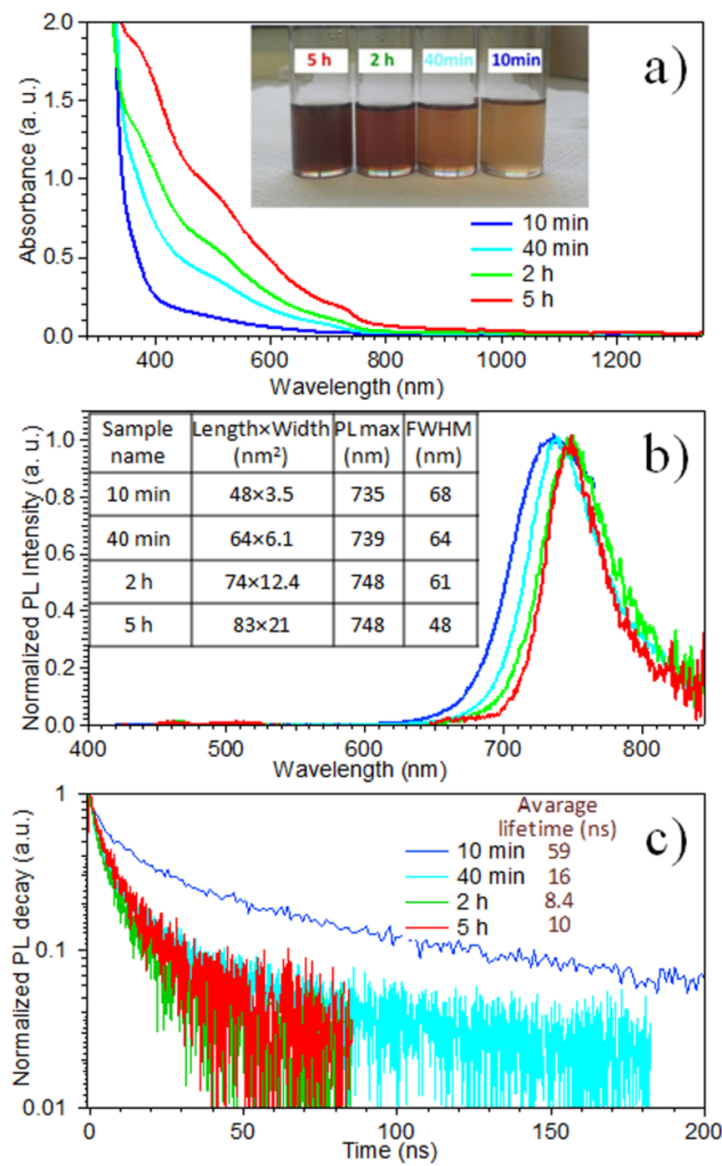

Figure 5. (a) Absorbance and (b) PL spectra of NPL samples grown for $10 \mathrm{~min}$ (blue line), $40 \mathrm{~min}$ (cyan line), $2 \mathrm{~h}$ (green line), and $5 \mathrm{~h}$ (red line) and dispersed in chloroform. Inset of (a): Photographs of the as-prepared PbS NPL dispersions in TCE. Inset of (b): table with main PL data. (c) PL decay traces of the different NPLs. The average lifetimes of the corresponding NPLs are shown in the inset.

To avoid oxidation of the PbS NPLs and the emergence of emission peaks at wavelengths longer than $1000 \mathrm{~nm}$ when purification is performed under ambient conditions (Figure S12), the purification was performed under inert atmosphere. This also strongly enhanced the main PL peak at $735 \mathrm{~nm}$ (Figure S12, NPLs synthesized for $10 \mathrm{~min}$ ). Accordingly, the PL peak around $735 \mathrm{~nm}$ is assigned to band-edge emission, and the emission emerging at longer wavelengths is due to surface defects.

While the PL in our samples occurs at wavelengths that are significantly shorter than other PbS NPLs, ${ }^{20-23}$ they are in agreement with results of Schliehe et al., who characterized 2D $\mathrm{PbS}$ sheets by using correlated confocal microscopy and measured a PL peak around $720 \mathrm{~nm} .{ }^{17}$ They also calculated the corresponding thickness with a particle-in-a-box approach by considering an optical bandgap of $1.72 \mathrm{eV}$ and assuming a high frequency dielectric constant of 18.4 for $\mathrm{PbS}$ and found $2.2 \mathrm{~nm}$, similar to the $1.8 \mathrm{~nm}$ measured here by HR-TEM. However, another theoretical model reported by Yang and Wise estimated an optical band gap of around $1.0 \mathrm{eV}$ for $2.2 \mathrm{~nm}$ thick PbS nanosheets. ${ }^{39}$ The discrepancies between different experimental and theoretical results could in part be due to the differences in crystal structure. Calculations of the bulk $\mathrm{PbS}$ band structure have shown that the reduced symmetry in orthorhombic $\mathrm{PbS}$ opens up the band gap, with theoretical values reaching up to $0.7 \mathrm{eV}$ (B27, FeB-type structure) or even $1.8 \mathrm{eV}$ (for B33, CrB/TlI-type structure), respectively, significantly larger than the $0.4 \mathrm{eV}$ observed in NaCl-type $\mathrm{PbS}^{40-42}$ Thus, it is clear that a detailed structural investigation with XRD and HR-TEM is needed to identify the PbS crystal phase, and calculations of the PbS NPL band gap should incorporate the resulting modifications to the band structure as well in order to reach a quantitative agreement.

The PL spectra show sharp peaks with a spectral position ranging from 735 to $748 \mathrm{~nm}$ depending on the lateral extension of the NPLs (Figure $5 \mathrm{~b}$ ). The full width at half-maximum (fwhm) of the PL spectra reduces from $68 \mathrm{~nm}(156 \mathrm{meV})$ for the $10 \mathrm{~min}$ aliquot to $48 \mathrm{~nm}(106 \mathrm{meV})$ for the $5 \mathrm{~h}$ aliquot, which is for all samples significantly narrower than for $0 \mathrm{D} \mathrm{PbS}$ QDs with similar emission energies. ${ }^{43,44}$ For comparison, 0D QDs that were synthesized following Hines and Scholes ${ }^{45}$ displayed a band-edge absorption peak at $715 \mathrm{~nm}$ and a PL peak at $868 \mathrm{~nm}$, with a fwhm of $131 \mathrm{~nm}(217 \mathrm{meV}$, Figure S13). The fwhm narrowing from OD QDs to 2D NPLs, equal to a factor of 1.4-2.0, is similar as what is observed for CdSe NPLs despite the substantially different band edge degeneracy. CdSe NPLs with a PL maximum at $543 \mathrm{~nm}$ show a fwhm of 14 $\mathrm{nm}(59 \mathrm{meV}$, Figure S14), whereas CdSe QDs emitting at 548 $\mathrm{nm}$ show a fwhm of $23 \mathrm{~nm}(95 \mathrm{meV}$ ) (Figure S14), yielding a fwhm ratio of $1.6: 1$. Also, the Stokes shift is considerably smaller for NPLs, with values of $18-20 \mathrm{~nm}$ (41-47 meV) compared to $153 \mathrm{~nm}(306 \mathrm{meV})$ for the QDs, again in line with the behavior of CdSe NPLs (Figure S14). Figure 5c plots the time-resolved PL decay. The traces can be fitted well using a multiexponential function, yielding an amplitude-weighted average lifetime varying from 59 to 10 ns (see Table S4 for the individual components of the fits). These values are about 2 orders of magnitude faster than in comparable $\mathrm{PbS} \mathrm{QDs}^{46,47}$ that display microsecond lifetimes (Figure S11). Recently, Izquierdo et al. reported on HgTe NPLs which emit at $880 \mathrm{~nm}$ (40 nm fwhm), with a similar fast lifetime of 50 ns. ${ }^{48}$ Other materials active in this NIR region, such as $\mathrm{CuInS}_{2}$ or $\mathrm{AgInS}_{2}$ QDs, have much longer PL lifetimes and broad spectra, 49,50 demonstrating that 2D NPLs possess a distinct advantage for all applications requiring fast exciton recombination or narrow emission.

\section{CONCLUSION}

A series of colloidal 2D PbS NPLs have been synthesized using a simple approach with PbOctdX as a single-source precursor. The choice of ligand, along with the reaction temperature and time, allows one to control the lateral NPL size and tune the inplane dimensions from the strong to the weak confinement regime. Structural analysis indicates an orthorhombic crystal phase in these highly anisotropic nanostructures. The absorbance spectra are strongly blue-shifted from the bulk band gap of $0.4 \mathrm{eV}$, due to the $1.8 \mathrm{~nm}$ thickness of the NPLs. A narrow PL spectrum with a line width down to $50 \mathrm{~nm}$, a strongly reduced Stokes shift of $18 \mathrm{~nm}$, and significantly enhanced PL decay rates all support the formation of 2D excitons with larger oscillator strength and reduced excitonphonon coupling compared to OD PbS QDs. As for Cd-based NPLs with emission across the visible spectrum, NIR emitting 2D PbS NPLs are promising building blocks for future solutionprocessed photonic and optoelectronic applications. 


\section{ASSOCIATED CONTENT}

\section{S Supporting Information}

The Supporting Information is available free of charge on the ACS Publications website at DOI: 10.1021/acs.chemmater.6b05111.

Scheme of NPL reaction mechanism, TEM images of different synthesis and tables summarizing the NPL lateral sizes and thicknesses, XRD pattern of PbOctdX, additional absorbance and fluorescence spectra of the different samples, optical spectra and TEM image of $\mathrm{PbS}$ QDs, and optical spectra of CdSe QDs and NPLs (PDF)

\section{AUTHOR INFORMATION}

\section{Corresponding Author}

*E-mail: iwan.moreels@iit.it.

\section{ORCID}

Ali Hossain Khan: 0000-0001-7155-0200

Iwan Moreels: 0000-0003-3998-7618

\section{Funding}

The present publication is realized with the support of the Ministero degli Affari Esteri e della Cooperazione Internazionale (IONX-NC4SOL). This project has also received funding from the European Union's Horizon 2020 research and innovation program under Grant Agreement No. 696656 (GrapheneCore1).

\section{Notes}

The authors declare no competing financial interest.

\section{REFERENCES}

(1) Lhuillier, E.; Robin, A.; Ithurria, S.; Aubin, H.; Dubertret, B. Electrolyte-gated colloidal nanoplatelets-based phototransistor and its use for bicolor detection. Nano Lett. 2014, 14, 2715-2719.

(2) Chen, Z. Y.; Nadal, B.; Mahler, B.; Aubin, H.; Dubertret, B. Quasi-2D colloidal semiconductor nanoplatelets for narrow electroluminescence. Adv. Funct. Mater. 2014, 24, 295-302.

(3) Lorenzon, M.; Christodoulou, S.; Vaccaro, G.; Pedrini, J.; Meinardi, F.; Moreels, I.; Brovelli, S. Reversed oxygen sensing using colloidal quantum wells towards highly emissive photoresponsive varnishes. Nat. Commun. 2015, 6, 6434.

(4) Guzelturk, B.; Kelestemur, Y.; Olutas, M.; Delikanli, S.; Demir, H. V. Amplified spontaneous emission and lasing in colloidal nanoplatelets. ACS Nano 2014, 8, 6599-6605.

(5) She, C.; Fedin, I.; Dolzhnikov, D. S.; Demortière, A.; Schaller, R. D.; Pelton, M.; Talapin, D. V. Low-threshold stimulated emission using colloidal quantum wells. Nano Lett. 2014, 14, 2772-2777.

(6) Grim, J. Q.; Christodoulou, S.; Stasio, F. D.; Krahne, R.; Cingolani, R.; Manna, L.; Moreels, I. Continuous-wave biexciton lasing at room temperature using solution-processed quantum wells. Nat. Nanotechnol. 2014, 9, 891-895.

(7) Naeem, A.; Masia, F.; Christodoulou, S.; Moreels, I.; Borri, P.; Langbein, W. Giant exciton oscillator strength and radiatively limited dephasing in two-dimensional platelets. Phys. Rev. B: Condens. Matter Mater. Phys. 2015, 91, 121302.

(8) Lhuillier, E.; Pedetti, S.; Ithurria, S.; Nadal, B.; Heuclin, H.; Dubertret, B. Two-dimensional colloidal metal chalcogenides semiconductors: synthesis, spectroscopy, and applications. Acc. Chem. Res. 2015, 48, 22-30.

(9) Tessier, M. D.; Javaux, C.; Maksimovic, I.; Loriette, V.; Dubertret, B. Spectroscopy of single CdSe nanoplatelets. ACS Nano 2012, 6, 6751-6758.

(10) Kunneman, L. T.; Tessier, M. D.; Heuclin, H.; Dubertret, B.; Aulin, Y. V.; Grozema, F. C.; Schins, J. M.; Siebbeles, L. D. A. Bimolecular auger recombination of electron-hole pairs in two- dimensional $\mathrm{CdSe}$ and $\mathrm{CdSe} / \mathrm{CdZnS}$ core/shell nanoplatelets. J. Phys. Chem. Lett. 2013, 4, 3574-3578.

(11) Joo, J.; Son, J. S.; Kwon, S. G.; Yu, J. H.; Hyeon, T. Lowtemperature solution-phase synthesis of quantum well structured CdSe nanoribbons. J. Am. Chem. Soc. 2006, 128, 5632-5633.

(12) Ithurria, S.; Dubertret, B. Quasi 2D colloidal CdSe platelets with thicknesses controlled at the atomic level. J. Am. Chem. Soc. 2008, 130, 16504-16505.

(13) Li, Z.; Qin, H.; Guzun, D.; Benamara, M.; Salamo, G.; Peng, X. Uniform thickness and colloidal-stable $\mathrm{CdS}$ quantum disks with tunable thickness: synthesis and properties. Nano Res. 2012, 5, 337351.

(14) Ithurria, S.; Talapin, D. V. Colloidal atomic layer deposition (cALD) using self-limiting reactions at nanocrystal surface coupled to phase transfer between polar and nonpolar media. J. Am. Chem. Soc. 2012, 134, 18585-18590.

(15) Tessier, M. D.; Spinicelli, P.; Dupont, D.; Patriarche, G.; Ithurria, S.; Dubertret, B. Efficient exciton concentrators built from colloidal core/crown $\mathrm{CdSe} / \mathrm{CdS}$ semiconductor nanoplatelets. Nano Lett. 2014, 14, 207-213.

(16) van Veggel, F. C. J. M. Near-infrared quantum dots and their delicate synthesis, challenging characterization, and exciting potential applications. Chem. Mater. 2014, 26, 111-122.

(17) Schliehe, C.; Juarez, B. H.; Pelletier, M.; Jander, S.; Greshnykh, D.; Nagel, M.; Meyer, A.; Foerster, S.; Kornowski, A.; Klinke, C.; Weller, $\mathrm{H}$. Ultrathin $\mathrm{PbS}$ sheets by two-dimensional oriented attachment. Science 2010, 329, 550-553.

(18) Bielewicz, T.; Dogan, S.; Klinke, C. Tailoring the height of ultrathin $\mathrm{PbS}$ nanosheets and their application as field-effect transistors. Small 2015, 11, 826-833.

(19) Dogan, S.; Bielewicz, T.; Lebedeva, V.; Klinke, C. Photovoltaic effect in individual asymmetrically contacted lead sulfide nanosheets. Nanoscale 2015, 7, 4875-4883.

(20) Bhandari, G. B.; Subedi, K.; He, Y.; Jiang, Z.; Leopold, M.; Reilly, N.; Lu, H. P.; Zayak, A. T.; Sun, L. Thickness-Controlled Synthesis of Colloidal PbS Nanosheets and Their ThicknessDependent Energy Gaps. Chem. Mater. 2014, 26, 5433-5436.

(21) Jiang, Z.; Bhandari, G. B.; Premathilaka, S. M.; Khan, S.; Dimick, D. M.; Stombaugh, C.; Mandell, A.; He, Y.; Lu, H. P.; Sun, L. Growth of colloidal $\mathrm{PbS}$ nanosheets and the enhancement of their photoluminescence. Phys. Chem. Chem. Phys. 2015, 17, 23303-23307.

(22) Zhang, H.; Savitzky, B. H.; Yang, J.; Newman, J. T.; Perez, K. A.; Hyun, B.-R.; Kourkoutis, L. F.; Hanrath, T.; Wise, F. W. Colloidal synthesis of $\mathrm{PbS}$ and $\mathrm{PbS} / \mathrm{CdS}$ nanosheets using acetate free precursors. Chem. Mater. 2016, 28, 127-134.

(23) Khan, S.; Jiang, Z.; Premathilka, S. M.; Antu, A.; Hu, J.; Voevodin, A. A.; Roland, P. J.; Ellingson, R. J.; Sun, L. Few-atom-thick colloidal $\mathrm{PbS} / \mathrm{CdS}$ core/shell nanosheets. Chem. Mater. 2016, 28, $5342-5346$

(24) Li, H.; Zhitomirsky, D.; Grossman, J. C. Tunable and Energetically Robust PbS Nanoplatelets for Optoelectronic Applications. Chem. Mater. 2016, 28, 1888-1896.

(25) DePuy, C. H.; King, R. W. Pyrolytic Cis Eliminations. Chem. Rev. 1960, 60, 431-457.

(26) Gammer, C.; Mangler, C.; Rentenberger, C.; Karnthaler, H. P. Quantitative local profile analysis of nanomaterials by electron diffraction. Scr. Mater. 2010, 63, 312-315.

(27) Pradhan, N.; Katz, B.; Efrima, S. Synthesis of high-quality metal sulfide nanoparticles from alkyl xanthate single precursors in alkylamine solvents. J. Phys. Chem. B 2003, 107, 13843-13854.

(28) Khan, A. H.; Ji, Q.; Ariga, K.; Das, B.; Sarma, D. D.; Acharya, S. Synthesis and metallic probe induced conductance of Au tipped ultranarrow $\mathrm{PbS}$ rods. Chem. Commun. 2011, 47, 8421-8423.

(29) Khan, A. H.; Pal, S.; Dalui, A.; Pradhan, J.; Sarma, D. D.; Acharya, S. Solution-Processed Freestanding Ultrathin Two-dimensional PbS Nanocrystals with Efficient and Highly Stable Dielectric Properties. Chem. Mater. 2017, 29, 1175-1182.

(30) Lewis, E. A.; McNaughter, P. D.; Yin, Z.; Chen, Y.; Brent, J. R.; Saah, S. A.; Raftery, J.; Awudza, J. A. M.; Malik, M. A.; O’Brien, P.; 
Haigh, S. J. In situ synthesis of PbS nanocrystals in polymer thin films from Lead(II) Xanthate and Dithiocarbamate complexes: Evidence for size and morphology control. Chem. Mater. 2015, 27, 2127-2136.

(31) Morrison, P. J.; Loomis, R. A.; Buhro, W. E. Synthesis and Growth Mechanism of Lead Sulfide Quantum Platelets in Lamellar Mesophase Templates. Chem. Mater. 2014, 26, 5012-5019.

(32) Momma, K.; Izumi, F. VESTA 3 for three-dimensional visualization of crystal, volumetric and morphology data. J. Appl. Crystallogr. 2011, 44, 1272-1276.

(33) Shamsi, J.; Dang, Z.; Bianchini, P.; Canale, C.; Stasio, F. D.; Brescia, R.; Prato, M.; Manna, L. Colloidal synthesis of quantum confined single crystal $\mathrm{Cs} \mathrm{PbBr}_{3}$ nanosheets with lateral size control up to the micrometer range. J. Am. Chem. Soc. 2016, 138, 7240-7243.

(34) Chen, D.; Gao, Y.; Chen, Y.; Ren, Y.; Peng, X. Structure Identification of Two-Dimensional Colloidal Semiconductor Nanocrystals with Atomic Flat Basal Planes. Nano Lett. 2015, 15, 44774482.

(35) Grzechnik, A.; Friese, K. Pressure-induced orthorhombic structure of PbS. J. Phys.: Condens. Matter 2010, 22, 095402.

(36) Petkov, V.; Moreels, I.; Hens, Z.; Ren, Y. PbSe quantum dots: Finite, off-stoichiometric, and structurally distorted. Phys. Rev. B: Condens. Matter Mater. Phys. 2010, 81, 241304.

(37) Bertolotti, F.; Dirin, D. N.; Ibáñez, M.; Krumeich, F.; Cervellino, A.; Frison, R.; Voznyy, O.; Sargent, E. H.; Kovalenko, M. V.; Guagliardi, A.; Masciocchi, N. Crystal symmetry breaking and vacancies in colloidal lead chalcogenide quantum dots. Nat. Mater. 2016, 15, 987-994.

(38) Newman, J. D. S.; Blanchard, G. J. Formation of Gold nanoparticles using amine reducing agents. Langmuir 2006, 22, 58825887.

(39) Yang, J.; Wise, F. W. Electronic States of Lead-Salt Nanosheets. J. Phys. Chem. C 2015, 119, 26809-26816.

(40) Zagorac, D.; Doll, K.; Schön, J. C.; Jansen, M. Ab initio structure prediction for lead sulfide at standard and elevated pressures. Phys. Rev. B: Condens. Matter Mater. Phys. 2011, 84, 045206.

(41) Demiray, F.; Berber, S. Ab initio investigation of B16(GeS), $\mathrm{B} 27(\mathrm{FeB})$ and $\mathrm{B} 33(\mathrm{CrB} / \mathrm{TlI})$ phases of lead chalcogenides. Phys. Scr. 2013, 88, 015603 .

(42) Wang, S.; Zhang, J.; Zhang, Y.; Alvarado, A.; Attapattu, J.; He, D.; Wang, L.; Chen, C.; Zhao, Y. Phase-Transition Induced Elastic Softening and Band Gap Transition in Semiconducting PbS at High Pressure. Inorg. Chem. 2013, 52, 8638-8643.

(43) Weidman, M. C.; Beck, M. E.; Hoffman, R. S.; Prins, F.; Tisdale, W. A. Monodisperse, Air-Stable PbS Nanocrystals via Precursor Stoichiometry Control. ACS Nano 2014, 8, 6363-6371.

(44) Moreels, I.; Justo, Y.; De Geyter, B.; Haustraete, K.; Martins, J. C.; Hens, Z. Size-tunable, bright, and stable PbS quantum dots: A surface chemistry study. ACS Nano 2011, 5, 2004-2012.

(45) Hines, M. A.; Scholes, G. D. Colloidal PbS Nanocrystals with size-tunable near-infrared emission: Observation of post-synthesis selfnarrowing of the particle size distribution. Adv. Mater. 2003, 15, 18441849.

(46) Clark, S. W.; Harbold, J. M.; Wise, F. W. Resonant Energy Transfer in PbS Quantum Dots. J. Phys. Chem. C 2007, 111, 73027305.

(47) Greben, M.; Fucikova, A.; Valenta, J. Photoluminescence quantum yield of $\mathrm{PbS}$ nanocrystals in colloidal suspensions. J. Appl. Phys. 2015, 117, 144306.

(48) Izquierdo, E.; Robin, A.; Keuleyan, S.; Lequeux, N.; Lhuillier, E.; Ithurria, S. Strongly Confined $\mathrm{HgTe} 2 \mathrm{D}$ Nanoplatelets as Narrow Near-Infrared Emitters. J. Am. Chem. Soc. 2016, 138, 10496-10501.

(49) Li, L.; Pandey, A.; Werder, D. J.; Khanal, B. P.; Pietryga, J. M.; Klimov, V. I. Efficient Synthesis of Highly Luminescent Copper Indium Sulfide-Based Core/Shell Nanocrystals with Surprisingly Long-Lived Emission. J. Am. Chem. Soc. 2011, 133, 1176-1179.

(50) Dai, M.; Ogawa, S.; Kameyama, T.; Okazaki, K.-i.; Kudo, A.; Kuwabata, S.; Tsuboie, Y.; Torimoto, T. Tunable photoluminescence from the visible to near-infrared wavelength region of non- stoichiometric $\mathrm{AgInS}_{2}$ nanoparticles. J. Mater. Chem. 2012, 22, 12851-12858. 